Thibodeaux, C. A., Kulkarni, V., Chang, W.-S., Neumann, O., Cao, Y., Brinson, B., ... Halas, N. J. (2014). Impurity-Induced Plasmon Damping in Individual Cobalt-Doped Hollow Au Nanoshells. The Journal of Physical Chemistry B. doi:10.1021/jp504467j

\title{
Impurity-Induced Plasmon Damping in Individual Cobalt-Doped Hollow Au Nanoshells
}

\author{
Christyn A. Thibodeaux ${ }^{1}$ \\ Vikram Kulkarni ${ }^{2}$ \\ Wei-Shun Chang ${ }^{3}$ \\ Oara Neumann ${ }^{1}$ \\ Yang $\mathrm{CaO}^{4}$ \\ Bruce Brinson ${ }^{3}$ \\ Ciceron Ayala-Orozco ${ }^{3}$ \\ Chih-Wei Chen ${ }^{4}$ \\ Emilia Morosan $3,4,5$ \\ Stephan Link ${ }^{1,3,5,6}$ \\ Peter Nordlander 1, 4, 5 \\ Naomi J. Halas 1, 2, 3, 4, 5 \\ ${ }^{1}$ Department of Electrical and Computer Engineering \\ ${ }^{2}$ Applied Physics Graduate Program \\ ${ }^{3}$ Department of Chemistry \\ ${ }^{4}$ Department of Physics and Astronomy \\ ${ }^{5}$ Rice Quantum Institute \\ ${ }^{6}$ Laboratory for Nanophotonics
}

Rice University, MS-378, 6100 Main Street, Houston, Texas 77005, United States 


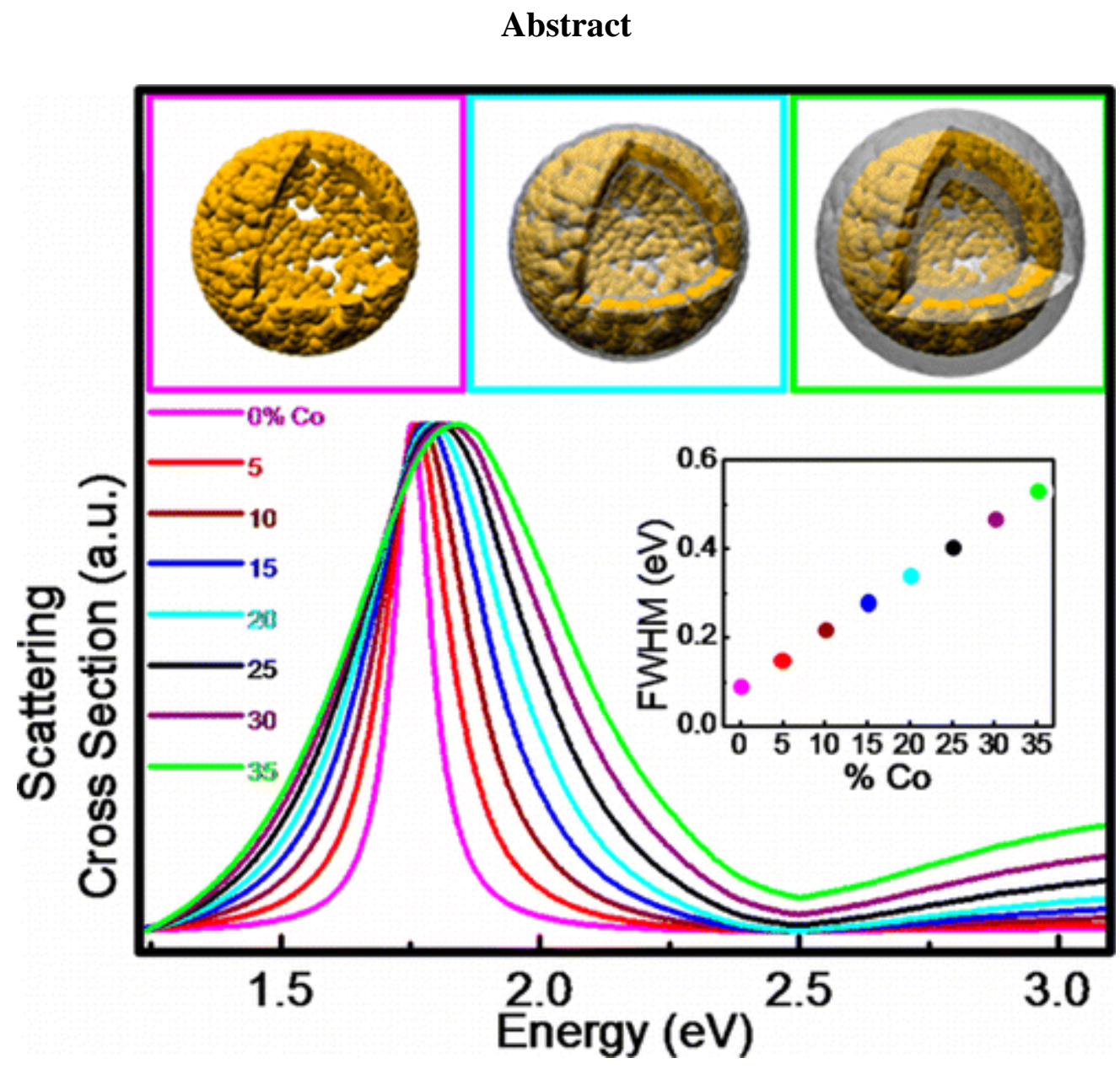

The optical properties of plasmonic nanoparticles in the size range corresponding to the electrostatic, or dipole, limit have the potential to reveal effects otherwise masked by phase retardation. Here we examine the optical properties of individual, sub-50 nm hollow $\mathrm{Au}$ nanoshells (Co-HGNS), where Co is the initial sacrificial core nanoparticle, using single particle total internal reflection scattering (TIRS) spectroscopy. The residual Co present in the metallic shell induces a substantial broadening of the homogeneous plasmon resonance line width of the Co-HGNS, where the full width at half-maximum (fwhm) broadens proportionately with increasing Co content. This doping-induced line broadening provides a strategy for controlling plasmon line width independent of nanoparticle size, and has the potential to substantially modify the relative decay channels for localized nanoparticle surface plasmons. 


\section{Introduction}

Plasmonic nanoparticles are currently of widespread interest as wavelength tunable nanoscale optical components, where both size and geometry provide important mechanisms for coupling to light. For spherical nanoparticles, an increase in nanoparticle size results in a redshifting of the dipole resonance and the appearance of additional plasmon resonances at higher frequencies. For nanoparticles of more complex geometries, plasmon resonances become a function of both size and shape. Conversely, nanoparticle size and geometry can be adjusted to maintain a constant plasmon resonance frequency in some plasmonic nanoparticles over a relatively large particle size range.(1)

While much attention has been paid to the tuning of plasmon resonance energies, far fewer studies have examined mechanisms for controlling nanoparticle plasmon line widths and line shapes. For the dipolar plasmon mode of a metallic nanosphere, the line width changes with increasing nanoparticle size, a characteristic signature of a bright plasmon mode. A direct comparison of the plasmon line shapes of individual nanorods and nanospheres showed the inherently narrower line shape characteristic of nanorods due to the absence of overlapping interband transitions.(2) For complex plasmonic clusters, the line shape broadening of superradiant plasmon modes can result in the energetic overlap of bright and dark plasmon modes, resulting in Fano resonant line shapes and the plasmonic analogue of electromagnetically induced transparency.(3-6)

For individual nanoparticles in the electrostatic limit, the homogeneous line width of a plasmon is inversely proportional to the plasmon lifetime. Plasmons have both radiative(7) and nonradiative(8-10) decay channels. For radiative decay, the plasmon decays into a photon.(11) For nonradiative decay, the plasmon decays by generating phonons and energetic electron-hole pairs.(12) Hot electron-hole pair generation due to plasmon decay can be used for optically generated carrier injection in photodetector devices,(13) has been directly shown to facilitate photocatalytic reactions on plasmonic substrates, $(14,15)$ and may facilitate DNA release at nanoparticle surfaces for light-triggered gene delivery.(16) Alteration of the plasmon line width of a nanoparticle is an indirect indication of a modification of its plasmon decay channels and may, for example, provide a route to more efficient hot carrier generation in specific plasmonic nanosystems.

Here we examine the effect of Co inpurities on the plasmon line shape of individual hollow gold nanoshells (Co-HGNS) with particle diameters of nominally $50 \mathrm{~nm}$. When HGNS are synthesized by a galvanic replacement reaction where a sacrificial core nanoparticle is oxidized simultaneously with the reduction of a metallic layer on the nanoparticle exterior, the resulting nanoparticle retains some of the residual metal of the initial sacrificial core nanoparticle.(17-19) While many studies have been reported on the ensemble optical properties of HGNS synthesized with a variety of sacrificial metal cores, very few single nanoparticle measurements have been performed, most notably surface enhanced Raman scattering (SERS) on individual HGNS nanoparticles.(20) The optical absorption of these nanoparticles has not been studied at the single particle level. This is primarily because of their small size. Single particle spectroscopies have generally been based on dark field illumination, where light scattered from the nanoparticle is detected, but nanoparticles of nominally $50 \mathrm{~nm}$ in diameter have small scattering cross sections and are difficult to observe by this approach, while hollow nanoparticles of this size are virtually undetectable. Alternative approaches to optically probe ultrasmall nanoparticles are being developed.(21) Here we employ total internal reflection 
scattering (TIRS) microscopy, which has an enhanced sensitivity to weakly scattering nanoparticles in this small size range.(22) At an angle greater than the critical angle, light is totally internally reflected at the surface of a sample and an evanescent wave is propagated along the surface. This evanescent wave can be scattered into free space by the nanoparticle, resulting in a high contrast signal with the absence of a signal from the substrate. With enhanced sensitivity using TIRS, particles as small as $20 \mathrm{~nm}$ can be resolved. The novelty of the TIRS system is the ability to isolate the NP scattering from the substrate scattering and to more efficiently excite the nanoparticles to provide better detection and resolution of particles with small scattering cross sections. By examining individual Co-HGNS particles, we observe that their dipolar plasmon line widths are sensitive to the presence of residual Co in the metallic layer. The line shape broadening scales linearly with the quantity of Co incorporated into the nanoparticle, and is well described by a straightforward theoretical model. This finding shows that impurities can provide a potential mechanism for controlling and tuning plasmon line shapes, which may find applications in tailoring plasmon decay channels for nanoparticles in this ultrasmall size range.

Co-HGNS were synthesized by coating cobalt $(\mathrm{Co})$ nanoparticles with gold (Au) in an $\mathrm{O}_{2}$ free environment, similar to previously published methods. $(23,24)$ Briefly, to a deaerated solution of $0.01 \mathrm{M}$ sodium citrate trihydrate $\left(\mathrm{Na}_{3} \mathrm{C}_{6} \mathrm{H}_{5} \mathrm{O}_{7} \cdot 3 \mathrm{H}_{2} \mathrm{O}\right)$ and $0.4 \mathrm{M}$ cobalt chloride hexahydrate $\left(\mathrm{CoCl}_{2} \cdot 6 \mathrm{H}_{2} \mathrm{O}\right), 1.0 \mathrm{M}$ sodium borohydride $\left(\mathrm{NaBH}_{4}\right)$ was added, and mixed using a mechanical stirrer (use of a magnetic stir bar induces magnetism and causes chain formation(25)). $\mathrm{NaBH}_{4}$ reduces the cobalt ions in solution:

$$
2 \mathrm{CoCl}^{2}+4 \mathrm{NaBH}_{4}+12 \mathrm{H}_{2} \mathrm{O} \rightarrow 2 \mathrm{Co}+4 \mathrm{NaCl}+14 \mathrm{H}_{2}+4 \mathrm{~B}(\mathrm{OH})_{3}
$$

and produces Co nanoparticles (CoNPs).(26) After allowing the reduction reaction to complete for 10 min under $\mathrm{N}_{2}$ flow, $33 \mathrm{~mL}$ of the CoNP solution is added to a $0.1 \mathrm{M}$ solution of chloroauric acid trihydrate $\left(\mathrm{HAuCl}_{4} \cdot 3 \mathrm{H}_{2} \mathrm{O}\right)$ in ambient air. As Co oxidizes, Au ions are reduced onto the CoNPs via galvanic replacement, since the reduction potential of gold is greater than the reduction potential of Co.(27)

Cobalt nanoparticles (CoNPs) are known to exhibit a strong field-dependent magnetization and to align themselves into (magnetic) dipole chains $(28,29)$ (Figure 1Ai). The Co-HGNS synthesis procedure prevents chain formation at the earliest stages by rapid vortexing; subsequent exposure of the solution during Au reduction to ambient air oxides the nanoparticle core, further reducing its magnetic response (Figure 1Aii, Aiii).(26, 30)

Transmission electron microscopy (TEM) images of the synthesized nanoparticles reveal a porous Au shell, allowing Co to diffuse out of the inner core region (Figure S1, Supporting Information).(27) The air/Co interface readily oxidizes while the Au shell grows over time. Since the $\mathrm{Au}$ shell grows rapidly at relatively high concentrations, a mixture of $\mathrm{Co}_{2} \mathrm{O}_{3}$ and $\mathrm{Co}$ is trapped in the Co-HGNS with larger shell thicknesses; residual $\mathrm{Co}_{2} \mathrm{O}_{3}$ also remains on the surface of the nanoshell and in solution (Figure S2, Supporting Information).(31) Multiple centrifugation cycles greatly reduce the presence of $\mathrm{Co}_{2} \mathrm{O}_{3}$ in solution but do not remove residual cobalt from inside the nanoshell. Variations in nanoparticle size from 20 to $100 \mathrm{~nm}$ can be obtained using this synthetic procedure by varying the reducing agent concentration. Energydispersive X-ray (EDX) measurements were obtained using a $200 \mathrm{kV}$ JEOL 2100f field emission TEM (Figure 1B). Correlating the TEM images with the EDX measurements reveals that Co:Au ratios obtained at the center of the nanoparticle vary with shell thickness. Elevated amounts of 
Co were consistently measured for nanoparticles consisting of thicker shell layers, which could possibly be attributed to a reduction in shell porosity. These measurements indicate that the volume fraction of $\mathrm{Co}$, ranging from 0 to $30 \%$, can be controlled by modifying shell thickness.
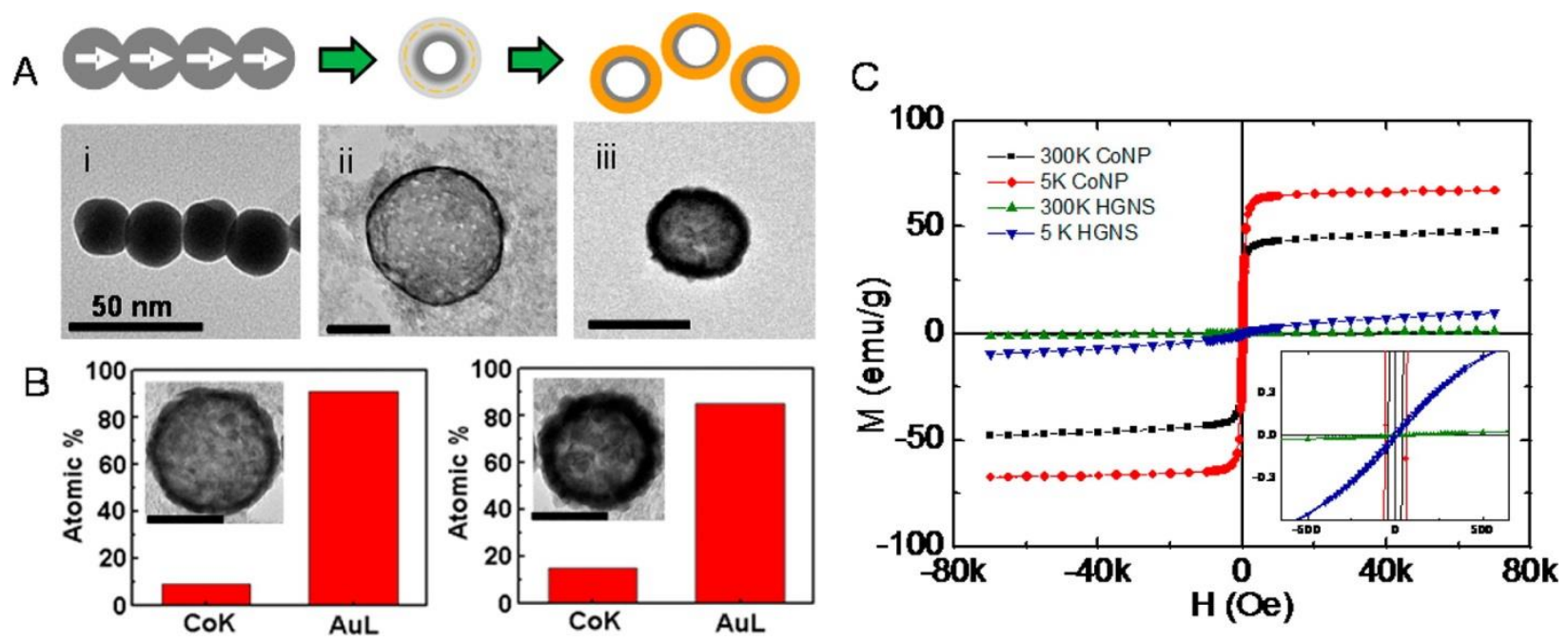

Figure 1. (A) The schematic of Co-HGNS synthesis: initially, rapid stirring disrupts magnetic chain formation of the nanoparticles, while galvanic replacement deposits a Au layer onto the sacrificial Co core. By exposing the Co core/Au shell nanoshells to ambient air, the core is oxidized, resulting in hollow gold shell nanostructures after centrifugation. Transmission electron microscopy (TEM) image of (i) a CoNP nanoparticle chain, (ii) oxidation of cobalt with the formation of a porous shell, and (iii) final Co-HGNS. (B) Energy Dispersive X-ray (EDX) and corresponding TEM images of Co-HGNS reveal differing Co:Au ratios for two particles with different gold shell thicknesses. (i) The thinner shell Co-HGNS corresponds to a lower level of residual Co, while (ii) the thicker shell retains a slightly higher level of Co. The scale bar for all TEM images is $50 \mathrm{~nm}$. (C) The field-dependent magnetization of CoNP at $T=300 \mathrm{~K}$ (black), CoNP at $T=5 \mathrm{~K}$ (red), Co-HGNS at $T=300 \mathrm{~K}$ (green), and CoHGNS at $T=5 \mathrm{~K}$ (blue) is measured. Magnetization of the synthesized nanoparticles is substantially reduced relative to the starting Co nanoparticles but not eliminated. Inset: magnification of the Co-HGNS hysteresis loop reveals a residual temperature dependent magnetization response.

Due to the presence of residual Co in the nanoparticles, we investigate the fielddependent magnetization properties of Co-HGNS. Field-dependent magnetization was measured at $T=5 \mathrm{~K}$ and $T=300 \mathrm{~K}$ (Figure 1C) (collected using a Quantum Design Magnetic Property Measurement System (QD MPMS, MPMS XL/Quantum Design, Inc.)). As expected for ferromagnetic nanoparticles, the magnetization for the CoNP displays hysteresis at both $T=5$ and $300 \mathrm{~K}$, and shows coercive field values of $\mathrm{HC}=60$ and $40 \mathrm{Oe}$, respectively. Both isotherms show a rapid increase of the magnetization with the applied field, indicating that the ferromagnetic ground state persists above $300 \mathrm{~K}$. The Co-HGNS display hysteresis at $T=5 \mathrm{~K}$, with the coercive field $\mathrm{HC}$ reduced to $10 \mathrm{Oe}$ satisfying superparamagnetic conditions. The corresponding magnetic field at $300 \mathrm{~K}$ is linear to $7 \mathrm{~T}$, indicating that the paramagnetic state for Co-HGNS has been reached at this temperature. These results show that the coercivity of CoHGNS is smaller than CoNP at the same temperature.(30)

By measuring the Co content using EDX at various positions on an individual nanoshell, the percentage of Co appears to be nearly the same $(\sim 20 \%)$, suggesting that the Co is homogeneously distributed throughout the nanoshell (Figure S3, Supporting Information). For this relatively high volume fraction of $\mathrm{Co}$, the weak magnetization signal verifies that a large 
portion of the residual $\mathrm{Co}$ is antiferromagnetic $\mathrm{Co}_{2} \mathrm{O}_{3}$. In order for Co to maintain its magnetic properties in ambient air, $\mathrm{Co}_{2} \mathrm{O}_{3}$ forms a self-terminating layer which serves as a protective barrier for the core to prevent further oxidation.(32) Since the presence of hysteresis verifies that the innermost core of the particle is magnetic $\mathrm{Co}$, we conclude the inner particle geometry is also composed of oxide. The final geometry likely consists of a magnetic Co core surrounded by $\mathrm{Co}_{2} \mathrm{O}_{3}$. This complex is coated by a porous $\mathrm{Au}$ shell, which allows excess $\mathrm{Co}_{2} \mathrm{O}_{3}$ to permeate through the shell.

Single particle scattering measurements were performed with a custom-built TIRS microscope (Figure 2A). Unpolarized light from a halogen lamp was focused using an oilimmersion dark-field condenser (Zeiss, N.A. = 1.40). Scattered light was collected by a $50 \times$ airspaced objective (Zeiss Epiplan-Neofluar, NA =0.8) and focused onto either an avalanche photodiode detector (APD, PicoQuant) or a CCD camera (Horiba Jobin Yvon). Due to a high incident angle of $67^{\circ}$ (larger than the critical angle of $41^{\circ}$ at the glass-air interface), the excitation in the total internal reflection geometry greatly reduces background scattered light and significantly increases the scattering field. Scattering images of the nanoparticles were acquired by scanning the sample with an $X Y$ piezo scanning stage (Physik Instrumente) across a $50 \mu \mathrm{m}$ pinhole located at the first image plane of the microscope. A LabView interface was designed to synchronize the stage movement and the data acquisition. Generally, the APD was used to image the sample. A typical image was composed of $128 \times 128$ pixels with an integration time of 5 $\mathrm{ms} /$ pixel. Single particle spectra were collected by guiding the light toward a liquid nitrogen cooled CCD camera attached to a spectrograph (Horiba Jobin Yvon). All measured single particle spectra were corrected for background scattering and normalized by the intensity of the incident light.

Co-HGNS were drop-cast onto a patterned TEM grid with a $30 \mathrm{~nm}$ layer of Formvar film (SPI Supplies) and were located and characterized by TEM initially, both to locate and to verify that isolated single nanoshells were being studied. The patterned TEM grid was then used to locate the same nanoshell under the optical microscope. The grid with particles facing outward was layered with a glass slide in contact with the dark field condenser (Figure 2A, inset). Indexmatching oil was used to ensure total internal reflection at the air-Formvar film interface. Images of three Co-HGNS with diameters of $\sim 40 \mathrm{~nm}$ recorded by TEM, TIRS microscopy, and conventional dark-field microscopy in a reflectance geometry are shown in Figure 2B. For identical integration times and detection conditions, scattering from the Co-HGNS detected with the TIRS microscope was not detected using the same microscope in a reflected light dark-field excitation configuration with an identical collection objective (NA of 0.8 for both TIR and reflected light dark-field geometries; NA $>0.94$ for dark-field excitation). Solid gold nanoparticles with an average diameter of $22 \mathrm{~nm}$ can even be detected with our TIRS microscope (Figure S5, Supporting Information). To further illustrate the higher detection sensitivity achieved with TIR vs reflected light dark-field excitation, Figure S6 (Supporting Information) shows SLR camera images obtained again under the same conditions (lamp intensity, collection NA, sample area, and camera integration time) for solid gold nanoparticles with a mean diameter of $70 \mathrm{~nm}$ excited using dark-field (Figure S6A) and TIR (Figure S6B) geometries. Nanoparticles, and more likely aggregates, are visible in both images, but single nanoparticles based on their green color are only detectable for TIR excitation for the selected integration time, illustrating that TIR excitation conditions are more sensitive. 


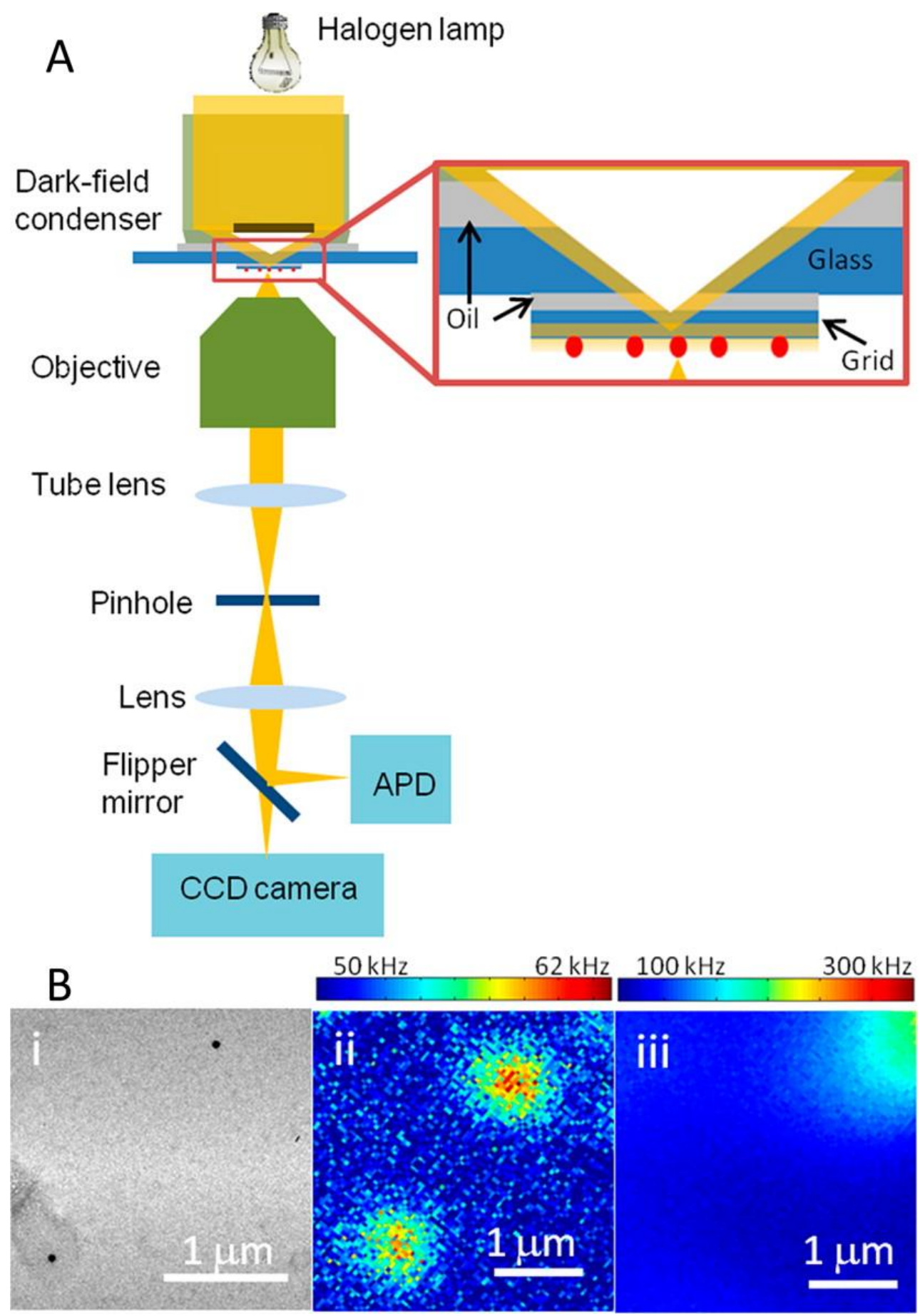

Figure 2. (A) Total internal reflection scattering (TIRS) spectroscopy system allowing high sensitivity for imaging small nanoparticles that are weak scatterers. Inset: a magnification of the TIR excitation condition for the multilayered sample with index matching oil. (B) Correlated electron and optical microscope images of the same areas: (i) TEM image, (ii) TIRS image, and (iii) reflected dark field image illustrating increased sensitivity obtained using TIRS. The bright spot in the upper right-hand corner of part iii is due to increased background scattering, which is suppressed in TIRS mode and allows for the detection of the nanoparticles in (ii). The background in the upper right corner in (iii) is likely due to the copper TEM grid, which is absent in (ii) because the light is incident from different sides in (ii) vs (iii) and hence background irregularities contribute differently depending on which side of the sample they are present. 
The scattering spectra of the LSPR dipolar mode of several individual Co-HGNS and Au nanospheres varying in size and Co content are shown in Figure 3. The Co-HGNS show significantly more broadening than Au nanospheres of similar size. For example, for $50 \mathrm{~nm}$ diameter nanoparticles, the Co-HGNS displays a full width at half-maximum (fwhm) amplitude of $0.44 \mathrm{eV}$, while the $\mathrm{Au}$ nanosphere has a fwhm of $0.27 \mathrm{eV}$ (Figure 3A). For nanoparticles with a diameter of $100 \mathrm{~nm}$, the Co-HGNS has a fwhm of $0.66 \mathrm{eV}$, while for the Au nanosphere a fwhm of only $0.33 \mathrm{eV}$ was observed (Figure 3B). While the spectral line widths vary significantly for the Co-HGNS sampled, only minimal variations in the mean position of the spectral maximum were observed.

The larger FWHMs of the Co-HGNS quite likely indicate plasmon damping related to the presence of Co impurities. A pair of HGNS, nearly identical in size at approximately $35 \mathrm{~nm}$, have a visibly noticeable variation in volume fraction of Co. As shown in Figure 3C, the particle with the greater volume of $\mathrm{Co}$ (magenta) results in a broader line width, $0.36 \mathrm{eV}$, which is greater than that of the HGNS with less Co (green), $0.31 \mathrm{eV}$.

The broadening of the Co-HGNS can be explained by examining the dielectric functions of Au and Co directly (Figure S4, Supporting Information). The real part of the dielectric function is related to the polarization of the material, and will affect the peak position. Within the energy region of interest, the real parts of the dielectric functions of both materials are quite similar. Therefore, it is not surprising that the presence of Co does not shift the surface plasmon energy significantly. However, Co does have a noticeably larger imaginary component of the dielectric function in this energy range. Physically, the imaginary part of the dielectric function corresponds to absorption and to ohmic heating. Due to the greater absorption relative to pure $\mathrm{Au}$, it is possible that electron-hole generation may increase along with increased energy dissipation, corresponding to the plasmon peak broadening with increasing Co content.

The lifetime of the plasmon can be deduced by the homogeneous spectral width of the nanoparticle spectrum(2) where a broader spectral line width corresponds to a shorter plasmon lifetime. In the case of $\mathrm{Co}$, the enhanced absorption comes from pronounced interband transitions. These interband transitions result in the creation of electron-hole pairs and additional nonradiative decay channels, damping the resonance considerably. This process is also referred to as internal damping, where excited plasmons can, for example, decay into highly energetic electron-hole pairs due to a transfer of energy, resulting in SPR broadening.(33) 

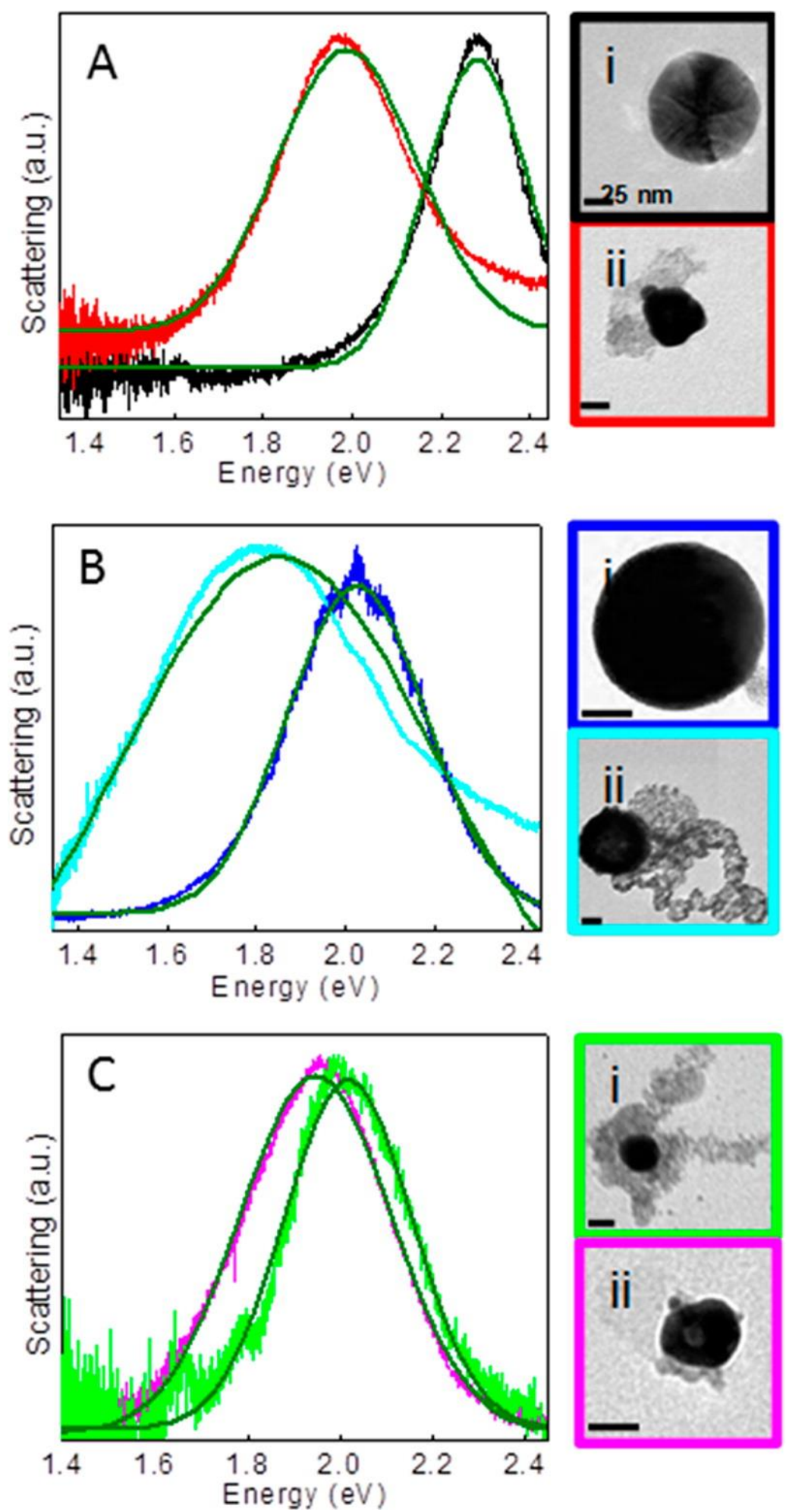

Figure 3. Experimental scattering spectra of (A) $50 \mathrm{~nm}$ Au colloid (black), $50 \mathrm{~nm}$ HGNS (red), (B) $100 \mathrm{~nm} \mathrm{Au}$ colloid (blue), $100 \mathrm{~nm}$ HGNS (cyan), and (C) $35 \mathrm{~nm}$ HGNS with greater (magenta) and smaller (green) volume fractions of Co were obtained using TIRS, shown with their corresponding TEM images. Each spectrum corresponds to a Gaussian function (dark green). 
To further investigate the increased damping due to the Co impurities, Mie theory simulations were performed. We model the structure as a hollow core with a mixed $\mathrm{Au}-\mathrm{Co}$ shell, embedded in a vacuum. The dielectric functions of $\mathrm{Au}$ and $\mathrm{Co}$ were obtained from refs 34 and 35. The Bruggeman formula is used to obtain an effective dielectric function of the shell.(36) Figure 4A shows the resulting normalized scattering cross section for a nanoshell of inner radius $30 \mathrm{~nm}$ and outer radius $33 \mathrm{~nm}$ with varying volume fractions of Co. The theoretical nanoshell dimensions were adjusted to keep a constant peak position of $\sim 2.23 \mathrm{eV}$. As the percentage of Co increases, the width of the scattering resonance also increases (Figure 4). The inset plots the exact fwhm of the scattering resonance as a function of Co percentage. This systematic broadening of the plasmon line shape with alloying is a property of the combination of $\mathrm{Au}$ and Co dielectric functions. In a recent study examining the effect of alloying in the Au-Ag alloyed hollow nanoshells using the same approach, no line shape broadening was observable as a function of metal composition.(1) Another important parameter that may influence the plasmon line shape, the presence of surface defects on the nanoparticle, was also analyzed in this study.(1) It was shown that, while multiple defects may induce a slight redshift of the plasmon resonance relative to a pristine geometry, the presence of multiple defects has virtually no contribution to line width broadening in the $\mathrm{Au}-\mathrm{Ag}$ hollow nanoshell case. On the basis of this study, we infer that the presence of surface defects in the $\mathrm{Au}-\mathrm{Co}$ hollow nanoshell would also not contribute to a broadening of the plasmon line shape.
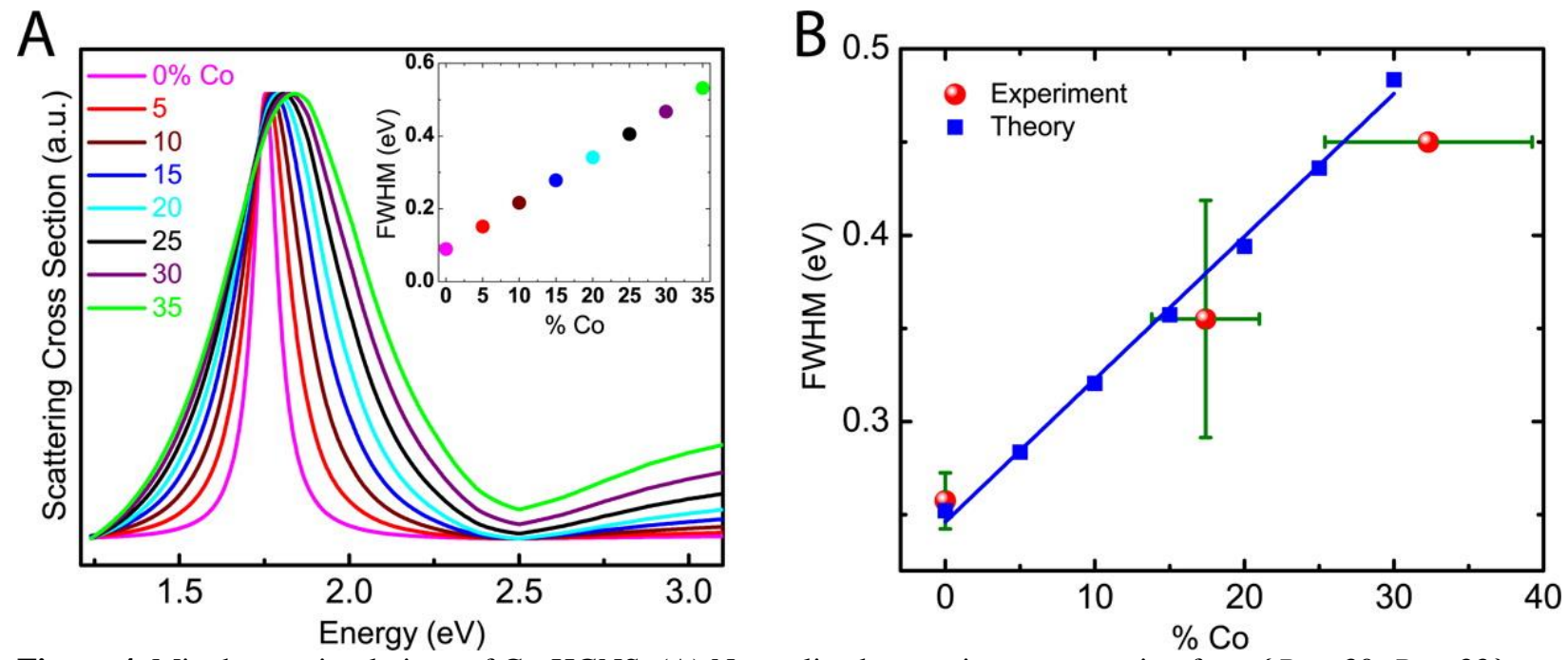

Figure 4. Mie theory simulations of Co-HGNS. (A) Normalized scattering cross section for a $\left\{R_{1}=30, R_{2}=33\right\} \mathrm{nm}$ Co-HGNS with varying percentages of Co, where $R_{1}$ is the inner radius and $R_{2}$ is the outer radius of the Co-HGNS. The inset shows the resulting full width at half-maximum. (B) Red, experimental scattering resonance full width at half-maxima as a function of Co percentage; blue, corresponding Mie theory. 
A comparison of the experimentally measured line widths and those obtained using Mie theory is shown in Figure 4B. Samples from three batches of nanoparticles with varying percentages of cobalt $(0,17.4$, and $32.3 \%)$ were analyzed using TEM for nanoparticle imaging, EDX for determining Co content of the candidate nanoparticles, and TIRS spectroscopy to obtain the scattering spectra. TEM initially confirmed a single particle of interest with the desired spherical geometry, to avoid nanoparticle chains or aggregates. The fwhm is plotted with respect to Co content for each single particle. Error bars show the observed fwhm variation in multiple single particles measured. The experimental results for $0 \%$ are actually solid Au nanospheres, since measurable Co impurities were present in all HGNS studied. The $32.3 \%$ Co case has no error bar in the $y$-direction because this Co concentration appears to be the maximum volume fraction of Co in a single particle and, as a result, only one particle was measured in this case (i.e., spherical nanoparticles were difficult to obtain in this Co impurity percentage range). The theoretical fwhm of mixed $\mathrm{Au}-\mathrm{Co}$ nanoshells are plotted in blue, and show good agreement with the experimental trends observed.

\section{Conclusion}

The presence of Co in HGNS nanoparticles in the nominally $50 \mathrm{~nm}$ diameter size range results in a significant broadening contribution to the homogeneous plasmon resonance line width. This broadening was identified in this study using TIRS spectroscopy on individual CoHGNS particles. TIRS offers high contrast images where even ultrasmall and weakly scattering particles are clearly detectable below the detection limits of dark field microspectroscopy. The impurity-induced plasmon damping observed here may provide a mechanism for increasing hot electron generation in plasmonic nanoparticles. This topic is of keen current interest for applications of plasmonic nanoparticles in photocatalysis. 


\title{
Supporting Information
}

Surface area measurements of the synthesized nanoparticles, transmission electron microscope images, XPS, energy-dispersive X-ray (EDX) analysis, TIRS, and scattering images of $\mathrm{Au}$ nanoparticles. This material is available free of charge via the Internet at http://pubs.acs.org.

The authors declare no competing financial interest.

\section{Acknowledgement}

This work was funded by the Robert A. Welch Foundation (grants C-1220, C-1222, and C-1664) and an ARO MURI (W911NF-12-1-0407). The authors are grateful for the TEM imaging and technical insight of Lisa Brown.

\author{
Abbreviations \\ HGNS hollow gold nanoshells \\ NIR near infrared \\ LSPR localized surface plasmon resonances \\ fwhm full width at half-maximum \\ TIR total internal reflection \\ TIRS total internal reflection scattering \\ SNR signal-to-noise ratio \\ TEM transmission electron microscopy \\ XPS X-ray photoelectron spectroscopy \\ EDX energy-dispersive X-ray \\ QD MPMS quantum design magnetic property Measurement System
}




\section{References}

1. Goodman, A. M.; Cao, Y.; Urban, C.; Neumann, O.; Ayala-Orozco, C.; Knight, M. W.; Joshi, A.; Nordlander, P.; Halas, N. J.The Surprising In Vivo Instability of Near-IR Absorbing Hollow Au-Ag Nanoshells ACS Nano 2014, 8, 3222- 3231

2. Sonnichsen, C.; Franzl, T.; Wilk, T.; von Plessen, G.; Feldmann, J.; Wilson, O.; Mulvaney, P.Drastic Reduction of Plasmon Damping in Gold Nanorods Phys. Rev. Lett. 2002, 88, 07740

3. Mirin, N. A.; Bao, K.; Nordlander, P.Fano Resonances in Plasmonic Nanoparticle Aggregates J. Phys. Chem. A 2009, 113, 4028- 4034

4. Fan, J. A.; Wu, C. H.; Bao, K.; Bao, J. M.; Bardhan, R.; Halas, N. J.; Manoharan, V. N.; Nordlander, P.; Shvets, G.; Capasso, F.Self-Assembled Plasmonic Nanoparticle Clusters Science 2010, 328, 1135- 1138

5. Urban, A. S.; Shen, X. S.; Wang, Y. M.; Large, N.; Wang, H.; Knight, M. W.; Nordlander, P.; Chen, H. Y.; Halas, N. J.Three-Dimensional Plasmonic Nanoclusters Nano Lett. 2013, 13, 4399- 4403

6. Lassiter, J. B.; Sobhani, H.; Fan, J. A.; Kundu, J.; Capasso, F.; Nordlander, P.; Halas, N. J.Fano Resonances in Plasmonic Nanoclusters: Geometrical and Chemical Tunability Nano Lett. 2010, 10, 3184- 3189

7. Lerme, J.; Baida, H.; Bonnet, C.; Broyer, M.; Cottancin, E.; Crut, A.; Maioli, P.; Del Fatti, N.; Vallee, F.; Pellarin, M.Size Dependence of the Surface Plasmon Resonance Damping in Metal Nanospheres J. Phys. Chem. Lett. 2010, 1, 2922- 2928

8. Kreibig, U.; Vollmer, M. Optical properties of metal clusters; Springer: Berlin, 1995; Vol. 25.

9. Hasegawa, M.; Watabe, M.Theory of Plasmon Damping in Metals. 1. General Formulation and Application to an Electron Gas J. Phys. Soc. Jpn. 1969, 27, 1393

10. Wang, X.; Zhang, Z. Y.; Hartland, G. V.Electronic Dephasing in Bimetallic Gold-Silver Nanoparticles Examined by Single Particle Spectroscopy J. Phys. Chem. B 2005, 109, 20324- 20330

11. Grigorchuk, N. I.Radiative Damping of Surface Plasmon Resonance in Spheroidal Metallic Nanoparticle Embedded in a Dielectric Medium J. Opt. Soc. Am. B 2012, 29, 3404- 3411

12. Ochoo, L.; Migwi, C.; Okumu, J., Damping Effect of the Inner Band Electrons on the Optical Absorption and Bandwidth of Metal Nanoparticles. J. Nanopart. Res. 2012, 14

13. Mubeen, S.; Lee, J.; Singh, N.; Kramer, S.; Stucky, G. D.; Moskovits, M.An Autonomous Photosynthetic Device in which all Charge Carriers Derive from Surface Plasmons Nat. Nanotechnol. 2013, 8, 247-251

14. Mukherjee, S.; Libisch, F.; Large, N.; Neumann, O.; Brown, L. V.; Cheng, J.; Lassiter, J. B.; Carter, E. A.; Nordlander, P.; Halas, N. J.Hot Electrons Do the Impossible: PlasmonInduced Dissociation of $\mathrm{H}_{2}$ on $\mathrm{Au}$ Nano Lett. 2013, 13, 240- 247

15. Lee, J.; Mubeen, S.; Ji, X. L.; Stucky, G. D.; Moskovits, M.Plasmonic Photoanodes for Solar Water Splitting with Visible Light Nano Lett. 2012, 12, 5014- 5019

16. Huschka, R.; Neumann, O.; Barhoumi, A.; Halas, N. J.Visualizing Light-Triggered Release of Molecules Inside Living Cells Nano Lett. 2010, 10, 4117-4122 
17. Sun, Y.; Mayers, B. T.; Xia, Y.Template-Engaged Replacement Reaction: A One-Step Approach to the Large-Scale Synthesis of Metal Nanostructures with Hollow Interiors Nano Lett. 2002, 2, 481- 485

18. Sun, Y.; Xia, Y.Alloying and Dealloying Processes Involved in the Preparation of Metal Nanoshells through a Galvanic Replacement Reaction Nano Lett. 2003, 3, 1569- 1572

19. Sun, Y.; Xia, Y.Mechanistic Study on the Replacement Reaction Between Silver Nanostructures and Chloroauric Acid in Aqueous Medium J. Am. Chem. Soc. 2004, 126, 3892- 3901

20. Schwartzberg, A. M.; Oshiro, T. Y.; Zhang, J. Z.; Huser, T.; Talley, C. E.Improving Nanoprobes using Surface-Enhanced Raman Scattering from 30-nm Hollow Gold Particles Anal. Chem. 2006, 78, 4732- 4736

21. Boyer, D.; Tamarat, P.; Maali, A.; Lounis, B.; Orrit, M.Photothermal Imaging of Nanometer-Sized Metal Particles Among Scatterers Science 2002, 297, 1160- 1163

22. Sonnichsen, C.; Geier, S.; Hecker, N. E.; von Plessen, G.; Feldmann, J.; Ditlbacher, H.; Lamprecht, B.; Krenn, J. R.; Aussenegg, F. R.; Chan, V. Z. H.; Spatz, J. P.; Moller, M.Spectroscopy of Single Metallic Nanoparticles using Total Internal Reflection Microscopy Appl. Phys. Lett. 2000, 77, 2949- 2951

23. Schwartzberg, A. M.; Olson, T. Y.; Talley, C. E.; Zhang, J. Z.Synthesis, Characterization, and Tunable Optical Properties of Hollow Gold Nanospheres J. Phys. Chem. B 2006, 110, 19935- 19944

24. Preciado-Flores, S.; Wang, D.; Wheeler, D. A.; Newhouse, R.; Hensel, J. K.; Schwartzberg, A.; Wang, L.; Zhu, J.; Barboza-Flores, M.; Zhang, J. Z.Highly Reproducible Synthesis of Hollow Gold Nanospheres with Near Infrared Surface Plasmon Absorption using PVP as Stabilizing Agent J. Mater. Chem. 2011, 21, 23442350

25. Xie, H. N.; Larmour, I. A.; Chen, Y. C.; Wark, A. W.; Tileli, V.; McComb, D. W.; Faulds, K.; Graham, D.Synthesis and NIR Optical Properties of Hollow Gold Nanospheres with LSPR Greater than one Micrometer Nanoscale 2013, 5, 765- 771

26. Glavee, G. N.; Klabunde, K. J.; Sorensen, C. M.; Hadjipanayis, G. C.Borohydride Reduction of Cobalt Ions in Water - Chemistry Leading to Nanoscale Metal, Boride, or Borate Particles Langmuir 1993, 9, 162- 169

27. Liang, H. P.; Wan, L. J.; Bai, C. L.; Jiang, L.Gold Hollow Nanospheres: Tunable Surface Plasmon Resonance Controlled by Interior-Cavity Sizes J. Phys. Chem. B 2005, 109, 7795- 7800

28. Stagg, S. M.; Knappenberger, K. L.; Dowgiallo, A. M.; Chandra, M.Three-Dimensional Interfacial Structure Determination of Hollow Gold Nanosphere Aggregates J. Phys. Chem. Lett. 2011, 2, 2946-2950

29. Lu, A. H.; Salabas, E. L.; Schuth, F.Magnetic Nanoparticles: Synthesis, Protection, Functionalization, and Application Angew. Chem., Int. Ed. 2007, 46, 1222- 1244

30. Salgueirino-Maceira, V.; Correa-Duarte, M. A.; Farle, M.; Lopez-Quintela, M. A.; Sieradzki, K.; Diaz, R.Synthesis and Characterization of Large Colloidal Cobalt Particles Langmuir 2006, 22, 1455-1458

31. Petitto, S. C.; Marsh, E. M.; Carson, G. A.; Langell, M. A.Cobalt oxide surface chemistry: The Interaction of $\mathrm{CoO}(100), \mathrm{Co}_{3} \mathrm{O}_{4}(110)$ and $\mathrm{Co}_{3} \mathrm{O}_{4}(111)$ with oxygen and water J. Mol. Catal. A: Chem. 2008, 281, 49- 58 
32. Hong, J. S.; Pyun, J.; Park, Y. W.; Kim, C. S.; Shim, I. B.Oxidation Effect in Cobalt Nanoparticles Magnetic Fluids IEEE Trans. Magn. 2009, 45, 2464- 2466

33. Aeschlimann, M. Electron Dynamics in Metallic Nanoparticles. Encyclopedia of Nanoscience and Nanotechnology; American Scientific Publishers: Stevenson Ranch, CA, 2004.

34. Johnson, P. B.; Christy, R. W.Optical Constants of the Noble Metals Phys. Rev. B 1972, $6,4370-4379$

35. Johnson, P. B.; Christy, R. W.Optical-Constants of Transition-Metals - Ti, V, Cr, Mn, Fe, Co, Ni, and Pd Phys. Rev. B 1974, 9, 5056-5070

36. Choy, T. C. Effective Medium Theory: Principles and Applications; Oxford University Press: Oxford, 1999 